I would like to thank Dr. E. Orowan for helpful discussions, and the British Iron and Steel Research Association, under the auspices of which the work was done, for permission to publish this paper.

Cavendish Laboratory, C. L. Sмith Cambridge. June 20.

${ }^{1}$ Orowan, E., Z. Phys., 89, 634 (1934).

2 Orowan, E., Proc. Phys. Soc., 52, 14 (1940).

'Andrade, E. N. da C., and Roscoe, R., Proc. Phys. Soc., 49, 152 (1937).

4 Dehlinger, U., and Gisen, F., Phy8. Z., 21, 862 (1934).

5 Taylor, G. I., Proc. Roy. Soc., A, 145, 362 (1934).

\section{Surface Tension and Detergency}

THE surface tension of an aqueous solution has long been recognized as one of the more important physical properties controlling its wetting and detergent powers, though early attempts to correlate these powers with equilibrium surface tension met with limited success. It is becoming generally appreciated that factors other than solute concentration influence surface tension, and that the condition of the surface must also be taken into account. Thus, the surface age involved in many detergent processes does not exceed a few seconds, and much of this time may be required for the establishment of diffusion equilibrium. For dilute solutions of longchain compounds the operative dynamic tension may vary over a range of 40 dynes/cm., and acknowledg. ment of the time required for surface equilibrium is clearly essential. A further factor, the change in equilibrium surface tension which occurs during change in area of a soluble film, has not previously been considered, but may prove to have considerable significance.

In very dilute solutions, the decrease in tension is proportional to solute concentration, and the Gibbs adsorption equation simplifies to the linear $\gamma-\Gamma$ relation $\gamma=\gamma_{0}-R T \Gamma$ typical of a gaseous film. Lateral cohesion, or repulsion between adsorbed molecules at higher concentrations, necessitates the introduction of correction factors; but these direct equations imply that the surface tension is dependent only on the surface excess $\Gamma$ so long as the surface is stationary (that is, of constant area). However, recent experiments ${ }^{1}$ have shown that the surface tension equivalent to a given surface excess per unit area of a long-chain adsorbate may be varied by as much as 10 dynes/cm. during expansion of the surface. If the adsorbed carbon chain is sufficiently long, the distribution of the forces between molecules at or near the surface (and thus the free surface energy) will depend upon the mean position of orientation of the adsorbed molecules. This orientation is likely to differ at stationary and expanding surfaces, and it is significant that the expansion effect is not apparent for short-chain solutes. Again, on contraction of a soluble film the migration of long-chain molecules away from the surface appears to take an appreciable time, so that a fresh contracting surface may contain an amount of adsorbed material in excess of the equilibrium amount.

Therefore, the surface tension of a solution, previously regarded as a function of bulk concentration and time only, must now be considered as subject to changes in area also, and the possible types of air-water interface may be subdivided as follows :
Dynamic

$(1) \rightarrow \begin{gathered}\text { Equilibrium } \\ \text { stationary }\end{gathered}$

$(2) \rightarrow \underset{\text { stationary }}{\text { Aged }}$

Dynamic expanding (4)

Equilibrium expanding (5)

Dynamic contracting $(6) \rightarrow$ Equilibrium contracting (7).

The term 'dynamic' is restricted, as already suggested in Nature $^{2}$, to surfaces at which the initial surface excess is in the process of accumulating. The term 'equilibrium' implies that the initial diffusion required in the accumulation of the surface excess has been completed, though secondary diffusion processes resulting from changes in surface area may still be operative. Arrows indicate those surface types which pass from one to another with time; (4) can only pass into (5) by increasing the solute concentration. A similar range of surface types exists for liquidliquid interfaces, though (3) is not well characterized.

The fresh surfaces operative in detergent processes are frequently expanding or contracting, and may fall into any one, or several, of the above types (except (3) ). For a given solution, the surface tensions corresponding to these different surface types vary widely, and it appears essential that the type of surface involved should be determined before any successful correlation between surface tension and detergent power can be achieved.

University College,

C. C. Adpison

Nottingham. July 29.

${ }^{1}$ Addison, J. Chem. Soc. (in the press).

${ }^{2}$ Addison, Nature, 156, 600 (1945).

\section{Optical Rotatory Power and Atomic Dimension}

IN a recent paper by Brauns ${ }^{1}$, an attempt is made to relate the differences in optical rotatory power of the respective alkyl halides and the differences in atomic dimensions of the halogens. The ratio of differences in specific rotation $(D$ line $)$ of the 2halogenopentanes were found to $\mathrm{be}(\mathrm{Br}, 41 \cdot 6-42 \cdot 6$, $\mathrm{Cl}):(\mathrm{I}, 46 \cdot 68-41 \cdot 6, \mathrm{Br})$, that is, $-0.86:+5 \cdot 08$. Atomic dimension differences require $(\mathrm{Br}-\mathrm{Cl})$ : $(\mathrm{I}-\mathrm{Br})=17 \cdot 1: 21 \cdot 4$, and on this basis the bromide should have a larger rotation than the chloride.

Whereas previous observations ${ }^{2}$ mentioned by Brauns had led to the conclusion that 2-chlorooctan $\theta,[\alpha]_{D}^{20^{\circ}} 35 \cdot 8^{\circ}$, had a higher rotation than the bromide (best estimated maximum, $[\alpha]_{D}^{20^{\circ}} 33 \cdot 8^{\circ}$ ), he now obtains 2-bromo-octane having $[\alpha]_{D}^{20^{\circ}} 40.6^{\circ}$, and providing a ratio of $17 \cdot 1: 30 \cdot 3$ for $(\mathrm{Br}-\mathrm{Cl})$ : $(\mathrm{I}-\mathrm{Br})$. In this observation he was preceded by Gerrard ${ }^{3}$.

This question of maximum rotation of halides becomes even more interesting in the example of phenylmethylcarbinol. Whereas $[\alpha]_{D} 50 \cdot 6^{\circ}$ for 1-chloro-1-phenylethane ${ }^{4}$ was for some time accepted as being near the maximum rotation, the rotatory power of the bromide being even less than this, Gerrard ${ }^{5}$ obtained a specimen of the chloride having $[\alpha]_{D}^{16^{\circ}} 93 \cdot 5^{\circ}$, by the interaction of the carbinol and phosphorus oxychloride in the presence of pyridine, and the bromide having $\alpha_{D}^{18^{\circ}} 131 \cdot 4^{\circ}(l=1)$ by means of phosphorus tribromide and pyridine. 